\title{
Topological entropy \\ of nonautonomous piecewise monotone dynamical systems on the interval
}

by Sergiř Koly a d a (Kiev), Michał M i s i u r e w i c z (Indianapolis, Ind.)
and Lubomír S no ha (Banská Bystrica)

Dedicated to the memory of Wiestaw Szlenk

\begin{abstract}
The topological entropy of a nonautonomous dynamical system given by a sequence of compact metric spaces $\left(X_{i}\right)_{i=1}^{\infty}$ and a sequence of continuous maps $\left(f_{i}\right)_{i=1}^{\infty}$, $f_{i}: X_{i} \rightarrow X_{i+1}$, is defined. If all the spaces are compact real intervals and all the maps are piecewise monotone then, under some additional assumptions, a formula for the entropy of the system is obtained in terms of the number of pieces of monotonicity of $f_{n} \circ \ldots \circ f_{2} \circ f_{1}$. As an application we construct a large class of smooth triangular maps of the square of type $2^{\infty}$ and positive topological entropy.
\end{abstract}

1. Introduction and main results. Let $X_{1, \infty}:=\left(X_{i}\right)_{i=1}^{\infty}$ be a sequence of compact metric spaces and $f_{1, \infty}:=\left(f_{i}\right)_{i=1}^{\infty}$ a sequence of continuous maps, where $f_{i}$ is a map from $X_{i}$ to $X_{i+1}$. For any positive integers $i, n$ set $f_{i}^{n}=f_{i+(n-1)} \circ \ldots \circ f_{i+1} \circ f_{i}$ and additionally $f_{i}^{0}=\operatorname{id}_{X_{i}}$. We also write $f_{i}^{-n}=\left(f_{i}^{n}\right)^{-1}$ (this notation will be applied to sets; we do not assume that the maps $f_{i}$ are invertible). We call $\left(X_{1, \infty} ; f_{1, \infty}\right)$ a nonautonomous discrete dynamical system. The trajectory of a point $x \in X_{1}$ is the sequence $\left(f_{1}^{n}(x)\right)_{n=0}^{\infty}$. We will speak of the $n$th iterate $\left(X_{1, \infty}^{[n]} ; f_{1, \infty}^{[n]}\right)$ of the system, where $X_{i}^{[n]}=X_{(i-1) n+1}$ and $f_{i}^{[n]}=f_{(i-1) n+1}^{n}$.

The topological entropy $h(f)$ of an autonomous dynamical system $(X ; f)$ was introduced by Adler, Konheim and McAndrew $[\mathrm{AKM}]$ and equivalent definitions were given by Bowen $[\mathrm{B}]$ and Dinaburg [D]. The topological entropy $h\left(f_{1, \infty}\right)$ of a nonautonomous dynamical system $\left(X_{1, \infty} ; f_{1, \infty}\right)$ was studied in [KS] under the additional assumption that all the spaces $X_{i}$ co-

1991 Mathematics Subject Classification: Primary 58F03; Secondary 58F08, 54H20.

Key words and phrases: nonautonomous dynamical system, topological entropy, triangular maps, piecewise monotone maps, $C^{\infty}$ maps. 
incide. In the present paper we generalize these definitions to the system $\left(X_{1, \infty} ; f_{1, \infty}\right)$.

The main aim of the paper is to prove analogues of the following result.

THEOREM 1.1 ([MS]). If $f$ is a piecewise monotone continuous selfmap of a compact interval and $c_{n}$ denotes the number of pieces of monotonicity of $f^{n}$ then

$$
h(f)=\lim _{n \rightarrow \infty} \frac{1}{n} \log c_{n} .
$$

We will consider a dynamical system $\left(I_{1, \infty} ; f_{1, \infty}\right)$, where $I_{i}$ is a compact real interval for any $i$. Moreover, we assume that every $f_{i}$ is piecewise monotone. By this we mean that there is a finite partition of $I_{i}$ into intervals such that $f_{i}$ is monotone (not necessarily strictly) on each element of this partition. Then $f_{1}^{n}$ is also piecewise monotone. A lap of a piecewise monotone map is any maximal (with respect to inclusion) interval on which it is monotone. We denote the number of laps of $f_{1}^{n}$ by $c_{1, n}$.

We want to find conditions under which the formula

$$
h\left(f_{1, \infty}\right)=\limsup _{n \rightarrow \infty} \frac{1}{n} \log c_{1, n},
$$

analogous to (1.1), holds. We present three sets of such conditions. In Theorem A, the assumptions are the weakest and easy to state. However, they are difficult to verify directly. Therefore we strengthen them to get weaker Theorems B and C, whose assumptions are often easy to verify.

Since the application in Section 5 uses Theorem B, this theorem can be perceived as the main result of the paper.

In the autonomous case compactness of the space plays an important role in the theory. In order not to lose this advantage, we have to put some restrictions on the behavior of our sequence of spaces.

Definition 1.2. A dynamical system $\left(I_{1, \infty} ; f_{1, \infty}\right)$ is said to be bounded if the lengths of the intervals are uniformly bounded from above.

To state Theorem B, we need the following definitions.

Definition 1.3. Let $\left(I_{1, \infty} ; f_{1, \infty}\right)$ be a dynamical system. It is said to have the Markov property if there exists $\delta>0$ and a sequence $\mathcal{C}_{1, \infty}$ of finite subsets $\mathcal{C}_{i} \subset I_{i}$ such that for every $i \geq 1$,

(a) the endpoints of $I_{i}$ belong to $\mathcal{C}_{i}$,

(b) either $f_{i}$ is monotone on the whole interval $I_{i}$ or the length of every component of $I_{i} \backslash \mathcal{C}_{i}$ is at least $\delta$,

(c) for every component $J$ of $I_{i} \backslash \mathcal{C}_{i}$ the map $f_{i}$ is monotone on $J$,

(d) $f_{i}\left(\mathcal{C}_{i}\right) \subset \mathcal{C}_{i+1}$. 
We use the name "Markov" because of the properties (a), (c) and (d). However, property (b) is also very important. While we do not want the elements of the Markov partitions to be too short (this holds automatically in the autonomous case), we admit exceptions if $f_{i}$ is globally monotone.

Definition 1.4. A bounded dynamical system $\left(I_{1, \infty} ; f_{1, \infty}\right)$ is said to be a Markov system if it has the Markov property and the maps $f_{i}, i=1,2, \ldots$, are equicontinuous.

Theorem B. If $\left(I_{1, \infty} ; f_{1, \infty}\right)$ is a Markov dynamical system then (1.2) holds.

Another important case when (1.2) holds is the following.

Definition 1.5. A bounded dynamical system $\left(I_{1, \infty} ; f_{1, \infty}\right)$ is said to be a finite piecewise monotone system if the set $\left\{f_{i}: i \in \mathbb{N}\right\}$ of maps has only finitely many distinct elements and each of them is piecewise monotone.

Theorem C. If $\left(I_{1, \infty} ; f_{1, \infty}\right)$ is a finite piecewise monotone system then (1.2) holds.

Note that Theorem 1.1 is a special case of Theorem C.

We deduce Theorems B and $\mathrm{C}$ from a more general, but more technical result.

Definition 1.6. A system $\left(I_{1, \infty} ; f_{1, \infty}\right)$, where all $f_{i}$ are piecewise monotone, is called long-lapped if there exists $\delta>0$ such that for every $i$ either $f_{i}$ is monotone or every lap of $f_{i}$ has length at least $\delta$. It will be called totally long-lapped if its $n$th iterate is long-lapped for every $n \geq 1$.

Theorem A. If $\left(I_{1, \infty} ; f_{1, \infty}\right)$ is a bounded totally long-lapped dynamical system then (1.2) holds.

Theorem A will be proved in Section 3. In Section 4 we show that Markov and finite systems are totally long-lapped, so Theorems B and C follow from Theorem A. We also present some related examples.

In Section 5 we apply Theorem B to triangular maps. A continuous map $F$ of the square $I \times I$ into itself is called triangular (see e.g. $[\mathrm{Kl}],[\mathrm{Ko}]$ ) if it is of the form

$$
F(x, y)=\left(f(x), g_{x}(y)\right) .
$$

It is said to be of type $2^{\infty}$ if it has periodic points of period $2^{n}$ for every $n \geq 0$ and of no other periods. Similarly to the case of continuous interval maps, if any other period is present, the topological entropy is positive, while if not all periods $2^{n}$ are present, the topological entropy is zero. However, the case of maps of type $2^{\infty}$ is different. While for the interval case they have zero entropy (see e.g. $[\mathrm{ALM}],[\mathrm{BC}]$ ), it is not necessarily the case for triangular maps (see $[\mathrm{Ko}]$ ). 
As an application of Theorem B we construct a large class of triangular maps of the square of type $2^{\infty}$ with positive topological entropy. Existence of such maps has been established in [Ko], but here we give a shorter proof based on Theorem B. In [BEL] an example of such a map of class $C^{r}$ was given for any finite $r$. We present a much more general construction, leading to a whole class of $C^{r}$ triangular maps of type $2^{\infty}$ with positive entropy (Theorem 5.7). Then we strengthen this result by showing that such maps exist even in the class $C^{\infty}$ (Theorem 5.8).

As we show after Theorem 5.8, for any map $F$ considered in Theorems 5.7 and 5.8 we have

$$
\limsup _{n \rightarrow \infty} \frac{1}{n} \log \operatorname{card} \operatorname{Fix}\left(F^{n}\right)=0,
$$

where $\operatorname{Fix}\left(F^{n}\right)$ is the set of fixed points of $F^{n}$. Moreover, there are no homoclinic points or horseshoes of any type. This is in contrast to $C^{0}$ interval maps (see e.g. $[\mathrm{ALM}],[\mathrm{BC}]$ ) and two-dimensional diffeomorphisms of class $C^{1+\varepsilon}([\mathrm{Ka}])$, where

$$
\limsup _{n \rightarrow \infty} \frac{1}{n} \log \operatorname{card} \operatorname{Fix}\left(F^{n}\right) \geq h(F),
$$

and if $h(F)>0$ then $F$ has homoclinic points and horseshoes (at least for some iterate).

Acknowledgements. This paper was written in part during the first and the third authors' visit to the Mathematisches Forschungsinstitut at Oberwolfach, Germany where they were supported by the VolkswagenStiftung (RiP-program at Oberwolfach), and the first author's visit to the Institut des Hautes Etudes Scientifiques at Bures-sur-Yvette, France. The third author was also partially supported by the Slovak grant agency, grant number $1 / 4015 / 97$. The second author was partially supported by the NSF grant DMS 9704422.

2. Topological entropy. For $i=1,2, \ldots$ let $\left(X_{i}, \varrho_{i}\right)$ be a compact metric space and let $f_{i}: X_{i} \rightarrow X_{i+1}$ be a continuous map. We are going to define the topological entropy of the system $\left(X_{1, \infty} ; f_{1, \infty}\right)$.

For each $n \geq 1$ the function

$$
\varrho_{1, n}(x, y)=\max _{0 \leq j \leq n-1} \varrho_{j+1}\left(f_{1}^{j}(x), f_{1}^{j}(y)\right)
$$

is a metric on $X_{1}$, equivalent to $\varrho_{1}$. A subset $E$ of $X_{1}$ is called $\left(n, f_{1, \infty}, \varepsilon\right)$ separated if for any two distinct points $x, y \in E, \varrho_{1, n}(x, y)>\varepsilon$. A set $F \subset X_{1}$ $\left(n, f_{1, \infty}, \varepsilon\right)$-spans another set $K \subset X_{1}$ if for each $x \in K$ there is $y \in F$ for which $\varrho_{1, n}(x, y) \leq \varepsilon$.

We define $s_{n}\left(f_{1, \infty}, \varepsilon\right)$ as the maximal cardinality of an $\left(n, f_{1, \infty}, \varepsilon\right)$-separated set and $r_{n}\left(f_{1, \infty}, \varepsilon\right)$ as the minimal cardinality of a set which $\left(n, f_{1, \infty}, \varepsilon\right)$-spans $X_{1}$. 
Similarly to $[\mathrm{KS}]$ where all spaces were the same, we define the topological entropy $h\left(f_{1, \infty}\right)$ of the system $\left(X_{1, \infty} ; f_{1, \infty}\right)$ by

$$
h\left(f_{1, \infty}\right)=\lim _{\varepsilon \rightarrow 0} \limsup _{n \rightarrow \infty} \frac{1}{n} \log s_{n}\left(f_{1, \infty}, \varepsilon\right)=\lim _{\varepsilon \rightarrow 0} \limsup _{n \rightarrow \infty} \frac{1}{n} \log r_{n}\left(f_{1, \infty}, \varepsilon\right) .
$$

The equality of the two limits can be proved very similarly to less general situations $[\mathrm{B}],[\mathrm{KS}]$, so we omit the proof.

It is also possible to provide an equivalent definition analogous to the original one from $[\mathrm{AKM}]$, using open covers. The only difference is that one has to use sequences of open covers of the spaces $X_{i}$ with Lebesgue numbers bounded away from 0 . However, in the present paper we are not going to use this definition, so we leave this easy task to the reader.

Although we keep using the traditional name "topological entropy," its value can depend on the metrics $\varrho_{1, \infty}$. This is similar to the situation of one map on a noncompact space: if we change the metric to another one which is equivalent but not uniformly equivalent then the entropy can change. In our case a kind of noncompactness enters the scene when we let $i \rightarrow \infty$.

Note that the following three lemmas hold. The first two can be proved similarly to the corresponding results in $[\mathrm{KS}]$ dealing with the case when all the spaces $X_{i}$ coincide, so we omit their proofs. The third one is simply taken from $[\mathrm{KS}]$.

Lemma 2.1. Let $\left(X_{1, \infty} ; f_{1, \infty}\right)$ be a dynamical system, where the spaces $X_{i}$ are compact metric. Then $h\left(f_{1, \infty}^{[n]}\right) \leq n h\left(f_{1, \infty}\right)$ for every $n \geq 1$.

Lemma 2.2. Let $\left(X_{1, \infty} ; f_{1, \infty}\right)$ be a dynamical system, where the spaces $X_{i}$ are compact metric and the maps $f_{i}, i=1,2, \ldots$, are equicontinuous. Then $h\left(f_{1, \infty}^{[n]}\right)=n h\left(f_{1, \infty}\right)$ for every $n \geq 1$.

LEMma $2.3([\mathrm{KS}])$. Let $f_{1, \infty}$ be a sequence of continuous selfmaps of a compact metric space $X$ converging uniformly to $f$. Then $h\left(f_{1, \infty}\right) \leq h(f)$.

3. Piecewise monotone sequences of interval maps. In this section we prove the main technical result of the paper, Theorem A. Some of the main ideas of the proof from $[\mathrm{MS}]$ (cf. $[\mathrm{ALM}],[\mathrm{BC}]$ ) will be preserved.

From now on we consider a dynamical system $\left(I_{1, \infty} ; f_{1, \infty}\right)$, where $I_{i}$ is a compact real interval for any $i$. Moreover, we assume that every $f_{i}$ is piecewise monotone.

First of all we emphasize that the assumption that the system is bounded plays an important role in the theorem. For instance, for the system $\left(I_{1, \infty} ; f_{1, \infty}\right)$ where $f_{i}(x)=2 x$ and $I_{i}=\left[0,2^{i}\right]$ the equality from the theorem does not hold. In Section 4 we show that the assumption that the system is totally long-lapped cannot be replaced by the assumption that it is longlapped (Examples 4.1, 4.4). 
By a mono-partition of $I_{i}$ we will understand a finite partition of $I_{i}$ into intervals (of any type, possibly degenerate) such that $f_{i}$ is monotone on every element of this partition. Let $\mathcal{P}_{1, \infty}$ be a sequence of mono-partitions of $I_{1, \infty}$, i.e., $\mathcal{P}_{i}$ is a mono-partition of $I_{i}$ into intervals $P_{i, 1}, \ldots, P_{i, n_{i}}, i \geq 1$. We say that $\left(P_{1, j_{1}}, \ldots, P_{n, j_{n}}\right)$ is a chain of length $n$ if the set

$$
\bigcap_{i=1}^{n} f_{1}^{-(i-1)}\left(P_{i, j_{i}}\right)
$$

is nonempty. We denote the family of all such sets by $\mathcal{P}_{1}^{n}$. Thus, (3.1) gives us a one-to-one correspondence between chains and elements of $\mathcal{P}_{1}^{n}$. Clearly, $\mathcal{P}_{1}^{n}$ is a mono-partition for $\left(I_{1, \infty}^{[n]} ; f_{1, \infty}^{[n]}\right)$. For a partition $\mathcal{A}$ of an interval into subintervals we denote by esscard $\mathcal{A}$ the number of nondegenerate elements of $\mathcal{A}$. Note that

$$
\operatorname{esscard} \mathcal{A} \leq \operatorname{card} \mathcal{A} \leq 2 \operatorname{esscard} \mathcal{A}+1 .
$$

Therefore we can set

$$
H\left(f_{1, \infty}, \mathcal{P}_{1, \infty}\right)=\limsup _{n \rightarrow \infty} \frac{1}{n} \log \operatorname{esscard} \mathcal{P}_{1}^{n}=\limsup _{n \rightarrow \infty} \frac{1}{n} \log \operatorname{card} \mathcal{P}_{1}^{n} .
$$

For an interval $J$ we denote its length by $|J|$. A sequence $\mathcal{P}_{1, \infty}$ of monopartitions of $I_{1, \infty}$ is said to be a $\mathcal{U}$-sequence if there is $\delta>0$ such that for any $k \geq 1$, the length of any nondegenerate element of $\mathcal{P}_{k}$ is at least $\min \left(\delta,\left|I_{k}\right|\right)$.

Clearly, if $\mathcal{P}_{1, \infty}$ is a $\mathcal{U}$-sequence for a bounded system then the cardinalities of the partitions $\mathcal{P}_{k}$ are uniformly bounded.

Lemma 3.1. Let $\left(I_{1, \infty} ; f_{1, \infty}\right)$ be a bounded dynamical system and let $\mathcal{P}_{1, \infty}$ be a $\mathcal{U}$-sequence. Then

$$
h\left(f_{1, \infty}\right) \leq H\left(f_{1, \infty}, \mathcal{P}_{1, \infty}\right) \leq h\left(f_{1, \infty}\right)+\log 2 .
$$

Pro of. By the definition of a $\mathcal{U}$-sequence, there is $\varepsilon>0$ such that for any $k \in \mathbb{N}$, either the length of any nondegenerate element of $\mathcal{P}_{k}$ is larger than $\min \left(2 \varepsilon,\left|I_{k}\right|\right)$ or $\mathcal{P}_{k}$ contains only one nondegenerate element. Let $E \subset I_{1}$ be an $\left(n, f_{1, \infty}, \varepsilon\right)$-spanning set of minimal cardinality. Fix $x \in E$ and look at the points $y \in I_{1}$ for which $\varrho_{1, n}(x, y) \leq \varepsilon$. Then $\varrho_{j+1}\left(f_{1}^{j}(x), f_{1}^{j}(y)\right) \leq \varepsilon$ for $j=0, \ldots, n-1$. The $\varepsilon$-neighborhood of $f_{1}^{j}(x)$ intersects at most 2 nondegenerate elements of $\mathcal{P}_{j+1}$, so the number of nondegenerate elements of $\mathcal{P}_{1}^{n}$ to which $y$ may belong is at most $2^{n}$. Since for every $y \in I_{1}$ there is $x \in E$ with $\varrho_{1, n}(x, y) \leq \varepsilon$, we get esscard $\mathcal{P}_{1}^{n} \leq 2^{n}$ card $E$, that is,

Therefore

$$
\frac{1}{n} \log \operatorname{esscard} \mathcal{P}_{1}^{n} \leq \frac{1}{n} \log r_{n}\left(f_{1, \infty}, \varepsilon\right)+\log 2 .
$$

$$
H\left(f_{1, \infty}, \mathcal{P}_{1, \infty}\right) \leq h\left(f_{1, \infty}\right)+\log 2 .
$$


Now we take an arbitrary $\varepsilon$ and choose an $\left(n, f_{1, \infty}, \varepsilon\right)$-separated set $F \subset$ $I_{1}$ of maximal cardinality. Let us estimate how many elements of $F$ can belong to one element of $\mathcal{P}_{1}^{n}$. An element $A$ of $\mathcal{P}_{1}^{n}$ corresponds to a chain $\left(P_{1, j_{1}}, \ldots, P_{n, j_{n}}\right)$. If $x, y$ are two adjacent elements of $A \cap F$ then there is $j$ with $0 \leq j \leq n-1$ such that $\varrho_{j+1}\left(f_{1}^{j}(x), f_{1}^{j}(y)\right)>\varepsilon$ and $f_{1}^{j}(x), f_{1}^{j}(y)$ are two adjacent elements of $f_{1}^{j}(A \cap F)$. Since the system $\left(I_{1, \infty} ; f_{1, \infty}\right)$ is bounded, there is $M$ such that $\left|I_{i}\right|<M \varepsilon$ for every $i \in \mathbb{N}$. Hence, for each $j$ there are at most $M$ pairs of adjacent elements of $f_{1}^{j}(A \cap F)$ which are more than $\varepsilon$ apart. Hence, there are at most $n M$ pairs of adjacent elements of $A \cap F$, and therefore card $A \cap F \leq n M+1$. In such a way we get card $F \leq$ $(n M+1) \operatorname{card} \mathcal{P}_{1}^{n}$, that is,

$$
\frac{1}{n} \log s_{n}\left(f_{1, \infty}, \varepsilon\right) \leq \frac{1}{n} \log \operatorname{card} \mathcal{P}_{1}^{n}+\frac{1}{n} \log (n M+1) .
$$

Since $\lim _{n \rightarrow \infty}(1 / n) \log (n M+1)=0$, we get $h\left(f_{1, \infty}\right) \leq H\left(f_{1, \infty}, \mathcal{P}_{1, \infty}\right)$.

We need the following technical lemma (see [MS], [ALM]).

LEMmA 3.2. Let $\left(a_{n}\right)_{n=1}^{\infty}$ be a sequence of real numbers and let $b, u \in \mathbb{R}$, $p \in \mathbb{N}$ be such that

(a) $u>0$,

(b) $a_{n+1} \leq a_{n}+b$ for all $n$,

(c) if $n \geq p$ and $a_{n} / n \geq u$ then $a_{n+1} \leq a_{n}+u$.

Then $\lim \sup _{n \rightarrow \infty} a_{n} / n \leq u$.

We call an element $A$ of $\mathcal{P}_{1}^{n}$ and the corresponding chain $\left(P_{1, j_{1}}, \ldots, P_{n, j_{n}}\right)$ proper if $P_{n, j_{n}} \subset f_{1}^{n}(A)$. Denote the set of all proper elements of $\mathcal{P}_{1}^{n}$ by $\mathcal{R}_{n}$.

LEMMA 3.3. Let $\left(I_{1, \infty} ; f_{1, \infty}\right)$ be a bounded dynamical system and let $\mathcal{P}_{1, \infty}$ be a $\mathcal{U}$-sequence. Assume that $H\left(f_{1, \infty}, \mathcal{P}_{1, \infty}\right)>\log 3$. Then

$$
\limsup _{n \rightarrow \infty} \frac{1}{n} \log \operatorname{card} \mathcal{R}_{n}=H\left(f_{1, \infty}, \mathcal{P}_{1, \infty}\right) .
$$

Proof. Since the system $\left(I_{1, \infty} ; f_{1, \infty}\right)$ is bounded and $\mathcal{P}_{1, \infty}$ is a $\mathcal{U}$ sequence, there is $M>2$ such that card $\mathcal{P}_{i} \leq M$ for all $i$. If we can apply Lemma 3.2 to $a_{n}=\log \operatorname{card} \mathcal{P}_{1}^{n}, b=\log M$ and a number $u$ such that $\log 3<u<H\left(f_{1, \infty}, \mathcal{P}_{1, \infty}\right)$ then $\limsup _{n \rightarrow \infty} a_{n} / n \leq u<H\left(f_{1, \infty}, \mathcal{P}_{1, \infty}\right)$, a contradiction. Since conditions (a) and (b) of Lemma 3.2 are satisfied, (c) cannot be satisfied. Since $H\left(f_{1, \infty}, \mathcal{P}_{1, \infty}\right)>u$, we can find an arbitrarily large $n$ such that $a_{n} / n \geq u$. Therefore, we can find an arbitrarily large $n$ such that both $a_{n} / n \geq u$ and $a_{n+1}>a_{n}+u>a_{n}+\log 3$. In other words, we can find an arbitrarily large $n$ such that both $(1 / n) \log \operatorname{card} \mathcal{P}_{1}^{n} \geq u$ and $\operatorname{card} \mathcal{P}_{1}^{n+1}>3 \operatorname{card} \mathcal{P}_{1}^{n}$.

For any $i$, denote by $l_{i}$ the number of chains of length $i$ that can be completed to a chain of length $i+1$ (by attaching an element of $\mathcal{P}_{i+1}$ 
at the end) in at least 3 ways. Then for arbitrarily large $n$ we have both $(1 / n) \log \operatorname{card} \mathcal{P}_{1}^{n} \geq u$ and

$3 \operatorname{card} \mathcal{P}_{1}^{n}<\operatorname{card} \mathcal{P}_{1}^{n+1} \leq M l_{n}+2\left(\operatorname{card} \mathcal{P}_{1}^{n}-l_{n}\right)=2 \operatorname{card} \mathcal{P}_{1}^{n}+(M-2) l_{n}$.

Thus, we get $\exp (n u) \leq \operatorname{card} \mathcal{P}_{1}^{n}<(M-2) l_{n}$. Therefore, since $u$ can be chosen as close to $H\left(f_{1, \infty}, \mathcal{P}_{1, \infty}\right)$ as we wish,

$$
\limsup _{i \rightarrow \infty} \frac{1}{i} \log l_{i} \geq H\left(f_{1, \infty}, \mathcal{P}_{1, \infty}\right) .
$$

When we complete a chain of length $i$ to a chain of length $i+1$ and we can do it in at least 3 ways, then at least one of these chains of length $i+1$ is proper. Thus, card $\mathcal{R}_{i+1} \geq l_{i}$. Hence

$$
\limsup _{n \rightarrow \infty} \frac{1}{n} \log \operatorname{card} \mathcal{R}_{n}=\limsup _{n \rightarrow \infty} \frac{1}{n-1} \log \operatorname{card} \mathcal{R}_{n} \geq H\left(f_{1, \infty}, \mathcal{P}_{1, \infty}\right) .
$$

The opposite inequality follows from the fact that $\mathcal{R}_{n} \subset \mathcal{P}_{1}^{n}$.

LEMma 3.4. If $\mathcal{Q}$ and $\mathcal{S}$ are partitions of an interval I into intervals (possibly degenerate) and $\mathcal{P}$ is their common refinement then card $\mathcal{P} \leq$ $\operatorname{card} \mathcal{Q}+\operatorname{card} \mathcal{S}-1$.

We omit a simple proof, consisting of counting the number of places at which we have to cut the interval to obtain corresponding partitions.

LEMMA 3.5. Let $\mathcal{Q}_{1, \infty}$ and $\mathcal{S}_{1, \infty}$ be sequences of mono-partitions for a system $\left(I_{1, \infty} ; f_{1, \infty}\right)$. Assume that there is a constant $N$ such that card $\mathcal{Q}_{n} \leq$ $N$ and card $\mathcal{S}_{n} \leq N$ for every $n$. Then $H\left(f_{1, \infty}, \mathcal{S}_{1, \infty}\right)=H\left(f_{1, \infty}, \mathcal{Q}_{1, \infty}\right)$.

Pr o o f. For each $i$, let $\mathcal{P}_{i}$ be the common refinement of $\mathcal{Q}_{i}$ and $\mathcal{S}_{i}$. Then by Lemma 3.4, card $\mathcal{P}_{i}<2 N$. Let $\left(Q_{1, j_{1}}, \ldots, Q_{n, j_{n}}\right)$ be the chain corresponding to an element $J$ of $\mathcal{Q}_{1}^{n}$. Each $Q_{i, j_{i}}$ is partitioned into less than $2 N$ elements of $\mathcal{P}_{i}$. Since $f_{1}^{i}$ is monotone on $J$, the partition of $J$ into elements of $f_{1}^{-i}\left(\mathcal{P}_{i}\right)$ consists of less than $2 N$ elements, all of them intervals. The partition of $J$ into elements of $\mathcal{P}_{1}^{n}$ is the common refinement of $n$ such partitions. Hence, by Lemma 3.4, the cardinality of $\mathcal{P}_{1}^{n}$ restricted to $J$ is less than $2 n N$. Thus, card $\mathcal{P}_{1}^{n} \leq 2 n N$ card $\mathcal{Q}_{1}^{n}$, and therefore $H\left(f_{1, \infty}, \mathcal{P}_{1, \infty}\right)$ $\leq H\left(f_{1, \infty}, \mathcal{Q}_{1, \infty}\right)$. Since $\mathcal{P}_{1, \infty}$ is finer than $\mathcal{Q}_{1, \infty}$, we get $H\left(f_{1, \infty}, \mathcal{P}_{1, \infty}\right)$ $=H\left(f_{1, \infty}, \mathcal{Q}_{1, \infty}\right)$. Similarly, $H\left(f_{1, \infty}, \mathcal{P}_{1, \infty}\right)=H\left(f_{1, \infty}, \mathcal{S}_{1, \infty}\right)$. Therefore $H\left(f_{1, \infty}, \mathcal{S}_{1, \infty}\right)=H\left(f_{1, \infty}, \mathcal{Q}_{1, \infty}\right)$.

Let $f$ be piecewise monotone on $I$. Note that two adjacent laps of $f$ always overlap. We will call their intersection a turning interval (it can be a degenerate interval, consisting of one point). 
Lemma 3.6. A system $\left(I_{1, \infty} ; f_{1, \infty}\right)$ is long-lapped if and only if there exists a $\mathcal{U}$-sequence of partitions for this system.

P r o o f. If there exists a $\mathcal{U}$-sequence, the system is obviously long-lapped. Conversely, assume that it is long-lapped. For a given $n$, let $A$ be the set of the midpoints of turning intervals of $f_{n}$. As the elements of our partition of $I_{n}$ we take the elements of $A$ and the components of $I_{n} \backslash A$. The lengths of nondegenerate elements of this partition are at least $1 / 2$ of the lengths of the corresponding laps of $f_{n}$. Thus, since the system is long-lapped, the sequence of partitions which we constructed is a $\mathcal{U}$-sequence.

Recall that for a system $\left(I_{1, \infty} ; f_{1, \infty}\right)$ where all $f_{i}$ are piecewise monotone, we denoted by $c_{1, n}$ the number of laps of $f_{1}^{n}$. The analogous number for the $k$ th iterate of the system will be denoted by $c_{1, n}^{[k]}$. Thus, $c_{1, n}^{[k]}=c_{1, k n}$.

Lemma 3.7. If $\left(I_{1, \infty} ; f_{1, \infty}\right)$ is a bounded long-lapped dynamical system then

$$
\limsup _{n \rightarrow \infty} \frac{1}{n} \log c_{1, n}^{[k]}=k \limsup _{n \rightarrow \infty} \frac{1}{n} \log c_{1, n},
$$

and if $\mathcal{P}_{1, \infty}$ is a $\mathcal{U}$-sequence of partitions then

$$
H\left(f_{1, \infty}^{[k]}, \mathcal{P}_{1, \infty}^{[k]}\right)=k H\left(f_{1, \infty}, \mathcal{P}_{1, \infty}\right) .
$$

Proof. Let $\mathcal{P}_{1, \infty}$ be a $\mathcal{U}$-sequence of partitions for $\left(I_{1, \infty} ; f_{1, \infty}\right)$. Then, since the system $\left(I_{1, \infty} ; f_{1, \infty}\right)$ is bounded, there is $M$ such that card $\mathcal{P}_{i} \leq M$ for all $i$. Therefore if $0 \leq j \leq k-1$ then $c_{1, k n+j} \leq M^{k} c_{1, k n}$. Thus,

$$
\limsup _{i \rightarrow \infty} \frac{1}{i} \log c_{1, i}=\limsup _{n \rightarrow \infty} \frac{1}{k n} \log c_{1, k n}=\frac{1}{k} \limsup _{n \rightarrow \infty} \frac{1}{n} \log c_{1, n}^{[k]},
$$

and we get the second equality in a similar way.

Lemma 3.8. Let $\left(I_{1, \infty} ; f_{1, \infty}\right)$ be a bounded dynamical system and let $\mathcal{P}_{1, \infty}$ be a $\mathcal{U}$-sequence. Assume that $H\left(f_{1, \infty}, \mathcal{P}_{1, \infty}\right)>\log 3$. Then

$$
\limsup _{n \rightarrow \infty} \frac{1}{n} \log c_{1, n} \geq H\left(f_{1, \infty}, \mathcal{P}_{1, \infty}\right) .
$$

Proof. By Lemma 3.3 for every $\varepsilon>0$ there exists an arbitrarily large $n$ such that $\operatorname{card} \mathcal{R}_{n} \geq \exp \left(n\left(H\left(f_{1, \infty}, \mathcal{P}_{1, \infty}\right)-\varepsilon\right)\right)$. Thus, there exists an interval $B \in \mathcal{P}_{n}$ such that the number of elements $A \in \mathcal{P}_{1}^{n}$ for which $B \subset$ $f_{1}^{n}(A)$ is at least $\exp \left(n\left(H\left(f_{1, \infty}, \mathcal{P}_{1, \infty}\right)-\varepsilon\right)\right) /$ card $\mathcal{P}_{n}$. These intervals $A$ are disjoint and their images under $f_{1}^{n}$ contain the same interval. Hence, the number of laps of $f_{1}^{n}$ is larger than or equal to the number of these intervals. That is, we have 


$$
c_{1, n} \geq \exp \left(n\left(H\left(f_{1, \infty}, \mathcal{P}_{1, \infty}\right)-\varepsilon\right)\right) / \operatorname{card} \mathcal{P}_{n} .
$$

Since $\left(I_{1, \infty} ; f_{1, \infty}\right)$ is bounded, there is $M$ such that card $\mathcal{P}_{i} \leq M$ for all $i$. Hence, we can replace in (3.3) $\operatorname{card} \mathcal{P}_{n}$ by $M$. Thus,

$$
\limsup _{n \rightarrow \infty} \frac{1}{n} \log c_{1, n} \geq H\left(f_{1, \infty}, \mathcal{P}_{1, \infty}\right)-\varepsilon .
$$

Since $\varepsilon$ was arbitrary, we get (3.2).

Now we are ready to prove Theorem A.

Proof of Theorem A. By Lemma 3.6 there exists a $\mathcal{U}$-sequence $\mathcal{P}_{1, \infty}$ of partitions for $\left(I_{1, \infty} ; f_{1, \infty}\right)$. Then $f_{1}^{n}$ is monotone on every element of $\mathcal{P}_{1}^{n}$, and hence $\operatorname{card} \mathcal{P}_{1}^{n} \geq c_{1, n}$. Thus, by Lemma 3.1, we get

$$
\limsup _{n \rightarrow \infty} \frac{1}{n} \log c_{1, n} \leq h\left(f_{1, \infty}\right)+\log 2 .
$$

Replacing the system $\left(I_{1, \infty} ; f_{1, \infty}\right)$ by its $k$ th iterate and using Lemmas 2.1 and 3.7 , we get the same inequality with $\log 2$ replaced by $(1 / k) \log 2$. Since $k$ can be arbitrarily large, we get

$$
\limsup _{n \rightarrow \infty} \frac{1}{n} \log c_{1, n} \leq h\left(f_{1, \infty}\right) .
$$

Now we prove the opposite inequality. If $h\left(f_{1, \infty}\right)=0$ then clearly

$$
\limsup _{n \rightarrow \infty} \frac{1}{n} \log c_{1, n} \geq h\left(f_{1, \infty}\right)
$$

and we are done. Assume that $h\left(f_{1, \infty}\right)>0$. Then by Lemma 3.1, $H\left(f_{1, \infty}, \mathcal{P}_{1, \infty}\right)>0$, so there is $k$ such that $k H\left(f_{1, \infty}, \mathcal{P}_{1, \infty}\right)>\log 3$. By Lemma 3.7, $H\left(f_{1, \infty}^{[k]}, \mathcal{P}_{1, \infty}^{[k]}\right)>\log 3$. Although we do not know whether $\mathcal{P}_{1, \infty}^{[k]}$ is a $\mathcal{U}$-sequence, we can circumvent it. Since $\left(I_{1, \infty} ; f_{1, \infty}\right)$ is totally long-lapped, by Lemma 3.6 there exists a $\mathcal{U}$-sequence of partitions $\mathcal{Q}_{1, \infty}$ for $f_{1, \infty}^{[k]}$.

The system $\left(I_{1, \infty} ; f_{1, \infty}\right)$ (and therefore also its $k$ th iterate) are bounded, so there is $M$ such that card $\mathcal{P} \leq M$ and $\operatorname{card} \mathcal{Q} \leq M$ for every $n$. Hence, for every $n$ the cardinalities of $\mathcal{P}_{n}^{[k]}$ and $\mathcal{Q}_{n}$ are bounded by $N=M^{k}$. Thus, by Lemma 3.5 we get $H\left(f_{1, \infty}^{[k]}, \mathcal{P}_{1, \infty}^{[k]}\right)=H\left(f_{1, \infty}^{[k]}, \mathcal{Q}_{1, \infty}\right)>\log 3$. Thus, by Lemma 3.8 we get

$$
\limsup _{n \rightarrow \infty} \frac{1}{n} \log c_{1, n}^{[k]} \geq H\left(f_{1, \infty}^{[k]}, \mathcal{Q}_{1, \infty}\right)=H\left(f_{1, \infty}^{[k]}, \mathcal{P}_{1, \infty}^{[k]}\right) .
$$

In view of Lemmas 3.7 and 3.1, this yields

$$
\limsup _{n \rightarrow \infty} \frac{1}{n} \log c_{1, n} \geq H\left(f_{1, \infty}, \mathcal{P}_{1, \infty}\right) \geq h\left(f_{1, \infty}\right) .
$$


4. Markov systems and examples. The following example (and similarly Example 4.4) shows that in Theorem A the assumption that the system is totally long-lapped cannot be replaced by the assumption that it is long-lapped.

EXAMPLE 4.1. Let $I_{n}=I=[0,1]$ for every $n$. Take a sequence of points $0=x_{1}<x_{2}<\ldots$ converging to $1 / 2$. Now consider the sequence $f_{1, \infty}$ of continuous selfmaps of $I$ where for any $n$,

$$
f_{n}(x)= \begin{cases}0 & \text { if } x \in\{0,1\}, \\ x_{n} & \text { if } x \in\left\{x_{n}, 1-x_{n}\right\} \\ 1-x_{n} & \text { if } x=1 / 2 \\ \text { affine } & \text { in between }\end{cases}
$$

Since $f_{1, \infty}$ converges uniformly to the map

$$
f(x)= \begin{cases}x & \text { if } x \in[0,1 / 2] \\ 1-x & \text { if } x \in[1 / 2,1]\end{cases}
$$

and $h(f)=0$, by Lemma 2.3 we have $h\left(f_{1, \infty}\right)=0$. Every $f_{n}$ is a unimodal map and so $c_{1, n} \leq 2^{n}$. On the other hand, for every $n$ we have $f_{n}(0)=$ $f_{n}(1)=0$ and $f_{n}(I)=\left[0,1-x_{n}\right] \supset\left[0,1-x_{n+1}\right]$ and so $c_{1, n} \geq 2^{n}$. Hence $c_{1, n}=2^{n}$ and

$$
\limsup _{n \rightarrow \infty} \frac{1}{n} \log c_{1, n}=\log 2 .
$$

Since sometimes it may be difficult to verify whether a system is totally long-lapped using only the definition, in Section 1 we specified two special cases: Markov systems and finite piecewise monotone systems. It turns out that they are totally long-lapped.

LEMMA 4.2. Markov systems are totally long-lapped.

Proof. Assume that the system $\left(I_{1, \infty} ; f_{1, \infty}\right)$ is Markov. Fix $k \geq 1$. We have to prove that the system $\left(I_{1, \infty}^{[k]} ; f_{1, \infty}^{[k]}\right)$ is long-lapped. By the definition of the Markov property, $\left(I_{1, \infty} ; f_{1, \infty}\right)$ is long-lapped, i.e., there is $\delta>0$ such that for every $n$ either $f_{n}$ is monotone or every lap of $f_{n}$ has length at least $\delta$. Set $g_{i}=f_{(n-1) k+1}^{i}$ for $i \geq 0$. By equicontinuity, there is $\varepsilon>0$ (independent of $n$ ) such that if $|a-b|<\varepsilon$ then $\left|g_{i}(a)-g_{i}(b)\right|<\delta$ for $i=0,1, \ldots, k-1$.

Let $J$ be a lap of $f_{n}^{[k]}$. If $f_{n k-j}$ is monotone for $j=0,1, \ldots, k-1$, then $f_{n}^{[k]}$ is monotone. If this is not the case, take the smallest $j$ such that $f_{n k-j}$ is not monotone. The lap $J$ contains an interval $(a, b)$ such that $g_{i}((a, b))$ is disjoint from $\mathcal{C}_{(n-1) k+1+i}$ for $i=0,1, \ldots, k-j-1$ and $(a, b)$ is the maximal interval with this property. Then for at least one of $i=0, \ldots, k-j-1$ we must have $g_{i}(a) \in \mathcal{C}_{(n-1) k+1+i}$. Therefore, by property (d) of Definition 1.3, $g_{k-j-1}(a) \in \mathcal{C}_{n k-j}$. Similarly, $g_{k-j-1}(b) \in \mathcal{C}_{n k-j}$. Since these two points are distinct, by property (b) of Definition 1.3 we have 
$\left|g_{k-j-1}(a)-g_{k-j-1}(b)\right| \geq \delta$, and therefore $|a-b| \geq \varepsilon$. Hence, $|J| \geq \varepsilon$. This proves that $\left(I_{1, \infty}^{[k]} ; f_{1, \infty}^{[k]}\right)$ is long-lapped.

Lemma 4.3. Finite piecewise monotone systems are totally long-lapped.

P r o o f. Clearly, a finite piecewise monotone system is long-lapped. Every iterate of such a system is also finite piecewise monotone, and therefore long-lapped. Hence, the original system is totally long-lapped.

Now, Theorem B follows from Theorem A and Lemma 4.2, while Theorem $\mathrm{C}$ follows from Theorem A and Lemma 4.3.

A system $\left(I_{1, \infty} ; f_{1, \infty}\right)$ is Markov if it is bounded, has the Markov property and the maps $f_{i}$ are equicontinuous. None of these can be removed from the definition of Markov systems if one wishes Theorem B to work for them. The system $\left(I_{1, \infty} ; f_{1, \infty}\right)$ where $f_{i}(x)=2 x$ and $I_{i}=\left[0,2^{i}\right]$ shows that we cannot remove the assumption that the system is bounded. Example 4.1 serves as a counterexample if we remove the Markov property. Finally, the next example shows that equicontinuity cannot be omitted.

EXAMPLE 4.4. Let $I_{n}=[0,1]$ for every $n$. Set $d_{n}=1 /(n+2), a_{n}=$ $1 / 2-d_{n}$ and $b_{n}=1 / 2+d_{n}$. Define

$$
f_{n}(x)= \begin{cases}0 & \text { if } x \in\{0,1\}, \\ 1 & \text { if } x=1 / 2, \\ d_{n} & \text { if } x \in\left\{a_{n}, b_{n}\right\}, \\ \text { affine } & \text { in between. }\end{cases}
$$

Then $c_{1, n}=2^{n}$ for every $n$, and thus $\lim \sup _{n \rightarrow \infty}(1 / n) \log \operatorname{card} c_{1, n}=\log 2$. All conditions of Definition 1.4 are satisfied except equicontinuity. The system is long-lapped, but it is easy to see that it is not totally long-lapped (this will follow also from the fact that the conclusion of Theorem A does not hold for this system).

Let us show that $h\left(f_{1, \infty}\right)=0$. Fix $\varepsilon>0$ and an integer $m>1 / \varepsilon$. Divide $[0,1]$ into $m$ intervals $A_{1}, \ldots, A_{m}$ of length less than $\varepsilon$ each. Take also an integer $k$ such that if $n>k$ then $2 d_{n}<\varepsilon$. Now we form the itinerary $\left(i_{n}(x)\right)_{n=0}^{\infty}$ of a point $x \in[0,1]$ in the following way. If $n \leq k$ then $i_{n}(x)=j$ if $f_{1}^{n}(x) \in A_{j}$. If $n>k$ then $i_{n}(x)=0$ if $f_{1}^{n}(x) \in\left[a_{n}, b_{n}\right], i_{n}(x)=m+1$ if $f_{1}^{n}(x) \in\left[0, d_{n}\right]$, and $i_{n}(x)=j$ if $f_{1}^{n}(x) \in A_{j} \backslash\left(\left[a_{n}, b_{n}\right] \cup\left[0, d_{n}\right]\right)$. Note that if $n>k$ and $i_{n}(x)>0$ then $i_{j}(x)=m+1$ for all $j>n$. Thus, if $n>k$ then the number of possible itinerary beginnings of length $n$ is at most $m^{k}(n-k+1)(m+1)$. Moreover, if $i_{j}(x)=i_{j}(y)$ then $\left|f_{1}^{j}(x)-f_{1}^{j}(y)\right|<\varepsilon$. Therefore

$$
r_{n}\left(f_{1, \infty}, \varepsilon\right) \leq m^{k}(n-k+1)(m+1) .
$$

Hence, $h\left(f_{1, \infty}\right)=0$. 
We conclude this section by noting that even if a system is bounded and totally long-lapped, the maps $f_{i}, i=1,2, \ldots$, do not have to be equicontinuous. For instance, one can take $f_{i}=x^{i}$ on $[0,1]$.

5. Application: triangular maps of type $2^{\infty}$ with positive topological entropy. As an application we are going to construct a large class of triangular maps of the square of type $2^{\infty}$ with positive topological entropy (on the interval, maps of type $2^{\infty}$ have zero topological entropy, see e.g. [ALM]). Existence of such maps has been established in [Ko], but here we give a shorter proof based on Theorem A. In [BEL] an example of such a map of class $C^{r}$ was given for any finite $r$. We present a much more general construction, leading to a whole class of triangular maps of type $2^{\infty}$, class $C^{r}$ and with positive entropy. Then we strengthen this result by showing that such maps exist even in the class $C^{\infty}$ (Theorem 5.8).

We start with a series of examples.

Example 5.1. Denote by $\tau$ the full tent map, i.e., $\tau(x)=1-|1-2 x|$. Set $\tau_{k}(x)=2^{-k} \tau(x)$ for $k=0,1,2, \ldots$ (in particular, $\tau_{0}=\tau$ ). Note that $\tau^{k} \circ \tau_{k}=\tau$ for every $k$ and hence

$$
\tau^{r} \circ \tau_{k}=\tau^{r-k+1} \quad \text { whenever } \quad r \geq k \geq 0 .
$$

Consider the sequence $\varphi_{1, \infty}$ of selfmaps of $I$ starting with

$$
\begin{aligned}
& \tau_{0}, \tau_{0}, \tau_{0}, \underline{\tau_{1}}, \tau_{0}, \tau_{0}, \tau_{0}, \underline{\tau_{2}}, \tau_{0}, \tau_{0}, \tau_{0}, \underline{\tau_{1}}, \tau_{0}, \tau_{0}, \tau_{0}, \underline{\tau_{3}}, \\
& \tau_{0}, \tau_{0}, \tau_{0}, \underline{\tau_{1}}, \tau_{0}, \tau_{0}, \tau_{0}, \underline{\tau_{2}}, \tau_{0}, \tau_{0}, \tau_{0}, \underline{\tau_{1}}, \tau_{0}, \tau_{0}, \tau_{0}, \underline{\tau_{4}}, \ldots
\end{aligned}
$$

(we have underlined all maps different from $\tau_{0}$ ) and defined by $\varphi_{n}=\tau_{i(n)-1}$, where $i(n)$ is 1 if $n$ is odd and is equal to the number of trailing zeros in the binary expansion of $n$ if $n$ is even.

Important moments when we look how the composition of our maps looks like are $2^{n}-1$. Therefore we introduce the notation $\Phi_{n}=\varphi_{1}^{2^{n}-1}$. By the definition, for all $n$ we have $\Phi_{n+1}=\Phi_{n} \circ \tau_{n-1} \circ \Phi_{n}$. We show by induction that

$$
\Phi_{n}=\tau^{a_{n}}
$$

where $a_{n}=2^{n-1}+n-1$. For $n=1$ we have $a_{1}=1$ and $\Phi_{1}=\tau$, so (5.2) holds. Assume now that it holds for some $n$. Then $\Phi_{n+1}=\tau^{a_{n}} \circ \tau_{n-1} \circ \tau^{a_{n}}$. Since $a_{n} \geq n-1$, we can use (5.1), and we get $\Phi_{n+1}=\tau^{2 a_{n}+1-(n-1)}=\tau^{a_{n+1}}$. This completes the proof of (5.2).

Now we can consider the system $\left(I_{1, \infty}, f_{1, \infty}\right)$ such that $I_{1}=I, I_{2}=$ $\varphi_{1}(I), I_{3}=\varphi_{2}\left(\varphi_{1}(I)\right), \ldots$, and $f_{1}=\varphi_{1}, f_{2}=\left.\varphi_{2}\right|_{I_{2}}, f_{3}=\left.\varphi_{3}\right|_{I_{3}}, \ldots$ We know that then $h\left(f_{1, \infty}\right)=h\left(\varphi_{1, \infty}\right)$. Moreover, the system $\left(I_{1, \infty}, f_{1, \infty}\right)$ (unlike $\left.\left(I_{1, \infty}, \varphi_{1, \infty}\right)\right)$ is evidently Markov. The number of pieces of monotonicity of 
$\varphi_{1}^{2^{n}-1}$ (and therefore of $f_{1}^{2^{n}-1}$ ) is $2^{a_{n}}$. Clearly,

$$
\limsup _{n \rightarrow \infty} \frac{1}{2^{n}-1} \log 2^{a_{n}}=\limsup _{n \rightarrow \infty} \frac{2^{n-1}+n-1}{2^{n}-1} \log 2=\frac{1}{2} \log 2,
$$

and therefore by Theorem B, $h\left(\varphi_{1, \infty}\right) \geq(1 / 2) \log 2$.

In $[\mathrm{Ko}]$ there is an example of a class of triangular maps $F=\left(f, g_{x}\right)$ of type $2^{\infty}$ with positive topological entropy. The entropy is positive due to the fact that $h\left(F ; I_{\alpha}\right)>0$ for some $\alpha$. The entropy $h\left(F ; I_{\alpha}\right)$ is in fact the entropy of the sequence of maps $g_{\alpha}, g_{f(\alpha)}, g_{f^{n}(\alpha)}, \ldots$ For one of the abovementioned triangular maps $F$ this sequence coincides with the sequence $\varphi_{1, \infty}$ from Example 5.1. Thus the long computation of $h\left(F ; I_{\alpha}\right)$ from [Ko] can be replaced by the one from Example 5.1, which is much shorter and simpler.

Now we modify Example 5.1 in order to make maps smooth. We introduce a class of maps $\mathcal{T}^{r}$ consisting of concave unimodal maps $f:[0,1] \rightarrow$ $[0,1]$ of class $C^{r}$ such that $f(0)=f(1)=0$ and $f(1 / 2)=1$.

Example 5.2. Choose maps $\gamma_{n} \in \mathcal{T}^{r}$. We set $\sigma_{n}=\lambda_{n} \gamma_{n}$ for some $\lambda_{n} \in(0,1]$, which we will define in a moment. Then we set $\varphi_{n}=\sigma_{i(n)-1}$, where $i(n)$ is defined as in Example 5.1. The numbers $a_{n}$ are also defined as in Example 5.1, and we set $\Phi_{n}=\varphi_{1}^{2^{n}-1}$. Then again, by the definition, for all $n$ we have $\Phi_{n+1}=\Phi_{n} \circ \sigma_{n-1} \circ \Phi_{n}$.

Now our construction starts to differ from the one from Example 5.1. Namely, instead of iterating $\tau$ like we did there, we use some sequence of maps $\eta_{1, \infty}$ from $\mathcal{T}^{r}$. Instead of $\tau^{k} \circ \tau_{k}=\tau$ (and instead of (5.1)) we will have

$$
\eta_{1}^{k-1} \circ \sigma_{k-1}=\eta_{a_{k}+1}
$$

and instead of (5.2),

$$
\Phi_{n}=\eta_{1}^{a_{n}} .
$$

We define $\eta_{n}$ together with $\lambda_{n}$ by induction. We start with $\eta_{1}=\gamma_{0}$ and $\lambda_{0}=1$. Now suppose that $\eta_{n} \in \mathcal{T}^{r}$ is defined for all $n \leq a_{k}$ (note that $a_{1}=1$ ) and $\lambda_{n}$ is defined for all $n<k-1$. We take as $\lambda_{k-1}$ the leftmost preimage of 1 under $\eta_{1}^{k-1}$ (since $k \leq a_{k}$, we use $\eta_{n}$ 's that are already defined). Then $\left.\eta_{1}^{k-1}\right|_{\left[0, \lambda_{k-1}\right]}$ is an increasing map from $\left[0, \lambda_{k-1}\right]$ onto $[0,1]$. As a composition of concave increasing maps it is also concave. Since $\sigma_{k-1}=\lambda_{k-1} \gamma_{k-1}$, it is easy to check that $\eta_{1}^{k-1} \circ \sigma_{k-1} \in \mathcal{T}^{r}$. Thus, (5.3) defines $\eta_{a_{k}+1} \in \mathcal{T}^{r}$. For $i=2, \ldots, a_{k+1}-a_{k}$ we set $\eta_{a_{k}+i}=\eta_{k-2+i}$ (cf. (5.5) below). Here we also use $\eta_{n}$ 's that are already defined, since $a_{k+1}-a_{k}=a_{k}-k+2$. Hence, the induction step is complete.

Now we have to prove (5.4). Again we use induction. If $n=1$ then $\Phi_{1}=\sigma_{0}=\eta_{1}^{1}$, so (5.4) holds. Assume now that it holds for some $n$. Then 
$\Phi_{n+1}=\eta_{1}^{a_{n}} \circ \sigma_{n-1} \circ \eta_{1}^{a_{n}}$. By the definition of $\eta_{1, \infty}$ we have

$$
\begin{aligned}
\eta_{1}^{a_{n}} \circ \sigma_{n-1} & =\left(\eta_{a_{n}} \circ \eta_{a_{n}-1} \circ \ldots \circ \eta_{n}\right) \circ\left(\eta_{1}^{n-1} \circ \sigma_{n-1}\right) \\
& =\left(\eta_{a_{n+1}} \circ \eta_{a_{n+1}-1} \circ \ldots \circ \eta_{a_{n}+2}\right) \circ \eta_{a_{n}+1} .
\end{aligned}
$$

Therefore $\Phi_{n+1}=\eta_{1}^{a_{n+1}}$. This completes the induction step.

Now we get $h\left(\varphi_{1, \infty}\right) \geq(1 / 2) \log 2$ as in Example 5.1.

We will need some additional estimates connected with Example 5.2.

Lemma 5.3. Let $\alpha_{n}$ be the number of $i \in\{1, \ldots, n\}$ for which $\eta_{i}=\gamma_{0}$ in Example 5.2. Then $\alpha_{m} / m \geq 1 / 4$ for all $m$.

Proof. It is easy to produce estimates of $\alpha_{a_{n}}$ by induction. First, by definition, we have $\eta_{1}=\gamma_{0}$, so $\alpha_{a_{1}}=1$. For $k=1$, (5.3) reads $\sigma_{0}=\eta_{2}$ ( $\eta_{1}^{0}$ is the identity), so also $\eta_{2}=\gamma_{0}$. Then $a_{2}-a_{1}=3-1=2$, so $\eta_{3}=$ $\eta_{a_{1}+2}=\eta_{1-2+2}=\eta_{1}=\gamma_{0}$. Thus, $\alpha_{a_{2}}=\alpha_{3}=3$. Now, if $k \geq 2$ then we get $\eta_{a_{k}+2}, \ldots, \eta_{a_{k+1}}$ by repeating some $\eta_{i}$ with $i \leq a_{k}$ (we take $a_{k+1}-a_{k}-1$ distinct $i$ 's). Among these $\eta_{i}$ 's there may be at most $a_{k}-\alpha_{a_{k}}$ maps different from $\gamma_{0}$, so at least $\left(a_{k+1}-a_{k}-1\right)-\left(a_{k}-\alpha_{a_{k}}\right)$ of them are equal to $\gamma_{0}$. This gives us

$$
\alpha_{a_{k+1}}-\alpha_{a_{k}} \geq\left(a_{k+1}-a_{k}-1\right)-\left(a_{k}-\alpha_{a_{k}}\right),
$$

so $\alpha_{a_{k+1}}-(k+1) \geq 2\left(\alpha_{a_{k}}-k\right)$. Since $\alpha_{a_{2}}-2=1$, by induction we get $\alpha_{a_{n}} \geq 2^{n-2}+n$ for all $n \geq 2$.

If $n \geq 2$ and $a_{n} \leq m<a_{n+1}$ then

$$
\frac{\alpha_{m}}{m} \geq \frac{\alpha_{a_{n}}}{a_{n+1}} \geq \frac{2^{n-2}+n}{2^{n}+n} \geq \frac{1}{4} .
$$

If $m=1,2$ then $\alpha_{m} / m=1>1 / 4$.

In the next example we construct a triangular map using maps from Example 5.2.

EXAMPLE 5.4. The main idea of the construction is the same as in [Ko]. The map is going to be of the form $F(x, y)=\left(f(x), g_{x}(y)\right)$, where on essential pieces $g_{x}$ is equal to the maps $\sigma_{n}$ from Example 5.2. As $f: I \rightarrow I$ we take a unimodal map of type $2^{\infty}$ without wandering intervals. In the construction we will use some well known combinatorial properties of such maps (see e.g. [CE], [dMvS], [Ko]). Let us list them.

For every $n$ there are $2^{n}$ disjoint intervals whose union $K_{n}$ is invariant for $f$. Each component of $K_{n}$ contains 2 components of $K_{n+1}$ and a periodic point of period $2^{n}$ between them. The intersection of all $K_{n}$ 's is an invariant Cantor set $K$. The critical point $c$ of $f$ belongs to $K$.

Thus, the set $K_{1}$ has 2 components. Denote the one that does not contain $c$ by $L_{1}$. Now $K_{2} \backslash L_{1}$ has 2 components and we denote the one that does not contain $c$ by $L_{2}$. We continue this construction and obtain a sequence 
of intervals $L_{n}$ that approach $c$ and lie alternately to the left and right of $c$. We denote by $M_{n}$ the gap between $L_{n}$ and $L_{n+2}$. Note that for each $n$ the periodic point $p_{n}$ closest to $c$, of period $2^{n-1}$, belongs to $M_{n}$. There is only one periodic orbit of period $2^{n-1}$ for every $n$.

The trajectory of $c$ passes through $L_{1}$ every two units of time (starting with $f(c)$ ), through $L_{2}$ every four units of time (starting with $f^{2}(c)$ ), and in general through $L_{k}$ every $2^{k}$ units of time, starting with $f^{2^{k-1}}(c)$. This means that $f^{j}(c) \in L_{i}$ if the number of trailing zeros in the binary expansion of $j$ is $i-1$.

Comparing this property with the description of $i(n)$ from Example 5.1, we see that we can apply Example 5.2 if we set $g_{x}=\sigma_{0}$ if $x \in L_{1}$ and $g_{x}=\sigma_{k-2}$ if $x \in L_{k}$ for $k>1$. Then $h(F) \geq(1 / 2) \log 2$.

On the other hand, if $g_{x} \equiv 0$ at one point $x$ of every periodic orbit of $f$, then the only periodic points of $F$ are of the form $(p, 0)$, where $p$ is a periodic point of $f$, and then $F$ is of type $2^{\infty}$.

We have not completed our construction yet. If there are points in $I$ outside the smallest interval containing $L_{1}$ and $L_{2}$, we set $g_{x}=\sigma_{0}$ there. More importantly, we have to define $g_{c}$ and $g_{x}$ for $x \in M_{n}$. The first part is very simple, namely we set $g_{c} \equiv 0$. The second part is slightly more complicated. We start by setting $g_{p_{n}} \equiv 0$ (so we get $F$ of type $2^{\infty}$ ). Now we have to fill the gaps between $p_{n}$ and the endpoints of $M_{n}$. Take an auxiliary function $\psi: \mathbb{R} \rightarrow[0,1]$ of class $C^{\infty}$ such that $\psi$ is 0 on $(-\infty, 0]$ and 1 on $[1, \infty)$. For $J=[a, a+\alpha]$ define $\psi_{J}^{+}, \psi_{J}^{-}: J \rightarrow[0,1]$ by

$$
\psi_{J}^{+}(a+t)=\psi_{J}^{-}(a+\alpha-t)=\psi(t / \alpha) .
$$

Now on the gaps at both sides of $p_{n}$ we define $g_{x}$ as the corresponding $\psi_{J}^{ \pm}(x) \cdot \sigma_{k}$, so that the maps agree at both endpoints of the gap with the previously defined ones.

In Example 5.2, $\lambda_{n}$ was defined as the leftmost preimage of 1 under $\eta_{1}^{n}$. Since each $\eta_{i}$ belongs to $\mathcal{T}^{r}$, we have

$$
\lambda_{n}=\left(\left.\eta_{1}\right|_{[0,1 / 2]}\right)^{-1} \circ\left(\left.\eta_{2}\right|_{[0,1 / 2]}\right)^{-1} \circ \ldots \circ\left(\left.\eta_{n}\right|_{[0,1 / 2]}\right)^{-1}(1) .
$$

Each $\left(\left.\eta_{i}\right|_{[0,1 / 2]}\right)^{-1}$ maps $[0,1]$ onto $[0,1 / 2]$ and it is convex since $\eta_{i}$ is concave. Therefore

$$
\left(\left.\eta_{i}\right|_{[0,1 / 2]}\right)^{-1}(x) \leq x / 2
$$

for each $x$. Hence, $\lambda_{n} \leq 2^{-n}$ for every $n$, that is, $\lambda_{n}$ decreases exponentially with $n$. Hence, $\sigma_{n}$ converges to 0 uniformly, and therefore $g_{x}$ converges to $g_{c} \equiv 0$ uniformly as $x \rightarrow c$. This proves continuity of $F$.

In order to specify the triangular map $F$ in Example 5.4 we have to choose the maps $f$ and $\gamma_{n}$. There is enormous freedom in doing that, so we can try to narrow the choice in order to get better properties of $F$. For 
instance, we can choose $f$ and $\gamma_{n}$ with negative Schwarzian derivative, and then $F$ has maps with negative Schwarzian derivative both in the base and in each fiber.

Another problem is to make $F$ as smooth as possible. First, choose $f$ and $\gamma_{n}$ of class $C^{r}$ for some fixed finite $r$. We need some estimates. We use the notation from Example 5.4.

Lemma 5.5. Assume that $f$ is of class $C^{3}$ and $f^{\prime \prime}(c) \neq 0$. Then there are constants $\delta, \zeta>0$ such that for every $n$ the distance of $p_{n}$ from each endpoint of $M_{n}$ is larger than $\zeta \delta^{n}$.

Proof. It is easy to check that our $f$ belongs to the class $\mathcal{U}^{2}$ defined in Chapter VI of [dMvS], and therefore we can use the results of that chapter.

From Theorem 2.1 of Chapter VI of [dMvS] it follows that there exist $\delta, \zeta_{1}>0$ such that for every $n$ the length of $M_{n}$ is larger than $\zeta_{1} \delta^{n}$.

Denote the common endpoint of $L_{n}$ and $M_{n}$ by $a_{n}$ and the common endpoint of $L_{n+2}$ and $M_{n}$ by $b_{n}$. Then denote by $J_{n}$ the interval with endpoints $c$ and $b_{n}$. Again by Theorem 2.1 of Chapter VI of [dMvS], the ratios $\left|M_{n}\right| /\left|J_{n}\right|$ and $\left|L_{n}\right| /\left|M_{n}\right|$ are bounded from above by a constant $\zeta_{2}$ independent of $n$.

The map $f^{2^{n-1}}$ is decreasing on $M_{n} \cup J_{n}$. It fixes $p_{n}$, maps $a_{n}$ to $b_{n}$ and $b_{n}$ to some point of $L_{n}$ (that is, beyond $a_{n}$, if we look from $p_{n}$ ). By Lemma 2.1 of Chapter VI of [dMvS], on $M_{n} \cup J_{n}$ we have $f^{2^{n-1}}=\psi_{1} \circ \psi_{2} \circ \psi_{3}$, where $\psi_{1}$ and $\psi_{3}$ have distortion bounded by a constant $\zeta_{3}$ independent of $n$ and $\psi_{2}$ is the quadratic map $\psi_{2}(x)=x^{2}$. By the chain rule, the distortion of $f^{2^{n-1}}$ on $M_{n}$ is bounded by $\zeta_{3}^{2}$ multiplied by the distortion of $\psi_{2}$ on $\psi_{3}\left(M_{n}\right)$. Since the only critical point of $\psi_{1} \circ \psi_{2} \circ \psi_{3}$ is $c$, we have $\psi_{3}(c)=0$. Thus, the distortion of $\psi_{2}$ on $\psi_{3}\left(M_{n}\right)$ is equal to $\left|\psi_{3}\left(M_{n} \cup J_{n}\right)\right| /\left|\psi_{3}\left(J_{n}\right)\right|$. This number is in turn bounded by $\zeta_{3}\left|M_{n} \cup J_{n}\right| /\left|J_{n}\right| \leq \zeta_{3}\left(\zeta_{2}+1\right)$. Hence, the distortion of $f^{2^{n-1}}$ on $M_{n}$ is bounded by a constant $\zeta_{4}$ independent of $n$.

The average of $\left|\left(f^{2^{n-1}}\right)^{\prime}\right|$ on $M_{n}$ is equal to $\left|f^{2^{n-1}}\left(M_{n}\right)\right| /\left|M_{n}\right|$. Since $f^{2^{n-1}}\left(M_{n}\right)$ is contained in $M_{n} \cup L_{n}$, it is less than or equal to $1+\zeta_{2}$. Therefore the maximum of $\left|\left(f^{2^{n-1}}\right)^{\prime}\right|$ on $M_{n}$ is less than or equal to $\zeta_{4}\left(1+\zeta_{2}\right)$. This gives us $\left|b_{n}-p_{n}\right| \leq \zeta_{4}\left(1+\zeta_{2}\right)\left|a_{n}-p_{n}\right|$ and $\left|a_{n}-p_{n}\right| \leq \zeta_{4}\left(1+\zeta_{2}\right)\left|b_{n}-p_{n}\right|$. Hence, both $\left|a_{n}-p_{n}\right|$ and $\left|b_{n}-p_{n}\right|$ are larger than or equal to $\zeta \delta^{n}$, where the constant $\zeta=\zeta_{1} /\left(1+\zeta_{4}\left(1+\zeta_{2}\right)\right)$ is independent of $n$.

We denote the $C^{r}$-norm of a function $g$ by $\|g\|_{r}$.

Lemma 5.6. Assume that in Example 5.4 the map $f$ is of class $C^{r}$ and $\gamma_{n} \in \mathcal{T}^{r}, n=0,1,2, \ldots$, the norms $\left\|\gamma_{n}\right\|_{r}$ are bounded uniformly in $n$, and 


$$
\liminf _{n \rightarrow \infty} \frac{1}{n} \log d_{n}>-\frac{1}{4 r} \log \gamma_{0}^{\prime}(0),
$$

where $d_{n}$ is the minimum of the distances of $p_{n}$ from the endpoints of $M_{n}$. Then $F$ is of class $C^{r}$.

Proof. The whole base interval is divided into countably many intervals and over each of them the map $F$ is of the form $\left(f(x), \varphi(x) \gamma_{n}(y)\right)$. The maps $f$ and $\gamma_{n}$ are of class $C^{r}$ and $\varphi$ is of class $C^{\infty}$, so $F$ is of class $C^{r}$ there. In order to show that it is of class $C^{r}$ everywhere, we have to check what happens where two such intervals meet (that is, at the points $p_{n}$ ) and where these intervals accumulate (that is, at $c$ ).

The map $\varphi$ can be defined on the whole real line by setting $\varphi(x)=0$ outside the interval mentioned in the preceding paragraph, and then it is of class $C^{\infty}$ everywhere. Therefore over the points $p_{n}$ all partial derivatives of the second component of $F$ up to order $r$ are 0 from both sides and they are continuous over $p_{n}$ from both sides. This leaves the problem of what happens when we approach $c$.

If a function is of the form $\varphi(x) \gamma_{n}(y)$ then its partial derivatives are of the form $\varphi^{(k)}(x) \gamma_{n}^{(l)}(y)$. Let us look at both factors as $x$ approaches $c$. On $L_{n}$ the function $\varphi$ is constant, so its derivatives are 0 . On the gaps where the functions $\psi_{J}^{ \pm}$were used in the construction, we have $\varphi=\lambda_{n} \cdot \psi_{J}^{ \pm}$. By (5.6) we get

$$
\sup _{J}\left|\left(\psi_{J}^{+}\right)^{(k)}\right|=\sup _{J}\left|\left(\psi_{J}^{-}\right)^{(k)}\right|=|J|^{-k} \sup _{[0,1]}\left|\psi^{(k)}\right| .
$$

In this formula, $J$ is the interval between $p_{i}$ and one of the endpoints of $M_{i}$, where $i=n$ or $n+2$. Hence, $\left|\varphi^{(k)}\right| \leq \lambda_{n} \cdot d_{i}^{-k} \sup _{[0,1]}\left|\psi^{(k)}\right|$. By Lemma 5.3, (5.7) and (5.8), we have $\lambda_{n} \leq\left(\left.\gamma_{0}\right|_{[0,1 / 2]}\right)^{-E(n / 4)}(1)$, where $E(\cdot)$ denotes the integer part. Therefore

$$
\limsup _{n \rightarrow \infty} \frac{1}{n} \log \lambda_{n} \leq-\frac{1}{4} \log \gamma_{0}^{\prime}(0) .
$$

By (5.9) and since $k \leq r$, we have

$$
\limsup _{n \rightarrow \infty} \frac{1}{n} \log d_{n}^{-k}<\frac{1}{4} \log \gamma_{0}^{\prime}(0) .
$$

Thus,

$$
\lim _{x \rightarrow c} \varphi^{(k)}(x)=0 .
$$

Note that (5.10) also holds for $k=0$.

Since the norms $\left\|\gamma_{n}\right\|_{r}$ are bounded uniformly in $n$, from (5.10) it follows that all partial derivatives of the second component of $F$ up to order $r$ go to 0 uniformly in $y$ as $x \rightarrow c$. Since we already know that they are continuous except possibly at the points of the fiber over $c$, it remains to prove that 
they are all 0 if $x=c$. However, this follows immediately by induction with respect to the order of differentiation with respect to $x$ and the mean value theorem.

The next theorem follows directly from Lemmas 5.5 and 5.6. It shows that for a very wide choice of maps $f$ and $\gamma_{n}$ the map $F$ from Example 5.4 is of class $C^{r}$ with finite $r$.

TheOREM 5.7. Assume that in Example 5.4 the map $f$ is of class $C^{r}$ with $3 \leq r<\infty$ and $f^{\prime \prime}(c) \neq 0$. Then there exists a constant $N$ such that if the functions $\gamma_{n} \in \mathcal{T}^{r}$, the norms $\left\|\gamma_{n}\right\|_{r}$ are bounded uniformly in $n$ and $\gamma_{0}^{\prime}(0)>N$ then the map $F$ is of class $C^{r}$.

If we want to get $F$ of class $C^{\infty}$ then the choice is not so wide, but the construction still works if we take the map from $[\mathrm{M}]$ on the base.

Theorem 5.8. Assume that in Example 5.4 the map $f$ is the $C^{\infty}$ map constructed in $[\mathrm{M}]$, the maps $\gamma_{n}$ are in $\mathcal{T}^{\infty}$ and for every finite $r$ their norms $\left\|\gamma_{n}\right\|_{r}$ are bounded uniformly in $n$ (although the bounds may depend on $r$ ). Then the map $F$ is of class $C^{\infty}$.

Proof. There are formulas in $[\mathrm{M}]$ for the distance of the point $p_{n}$ to the endpoints of $M_{n}$. They give estimates of $d_{n}$ from below by reciprocals of polynomials in $n$. Therefore

$$
\liminf _{n \rightarrow \infty} \frac{1}{n} \log d_{n}=0
$$

while by concavity $\gamma_{0}^{\prime}(0) \geq 2$. Therefore (5.9) holds for all $r$, and by Lemma 5.6 the map $F$ is of class $C^{r}$ for every finite $r$.

Note that for any map $F$ considered in Theorems 5.7 and 5.8 there is at most one periodic orbit of a given period (we may assume for simplicity that the base map has only one fixed point). Therefore

$$
\limsup _{n \rightarrow \infty} \frac{1}{n} \log \operatorname{card} \operatorname{Fix}\left(F^{n}\right)=0 .
$$

Moreover, any periodic point $x$ is a saddle. Its stable manifold is the fiber over this point. The image of the whole space intersects this fiber only at $x$, and therefore there are no homoclinic points. Similarly, there are no horseshoes of any type, even for iterates of $F$.

There is another way to prove that the topological entropy of $C^{\infty}$ maps from Theorem 5.8 is positive. One can proceed as follows. If $G$ is a $C^{1}$ function defined on $I$, denote the length of the graph $\Gamma_{G}$ of $G$ by $\operatorname{Vol}\left(\Gamma_{G}\right)$. For a $C^{\infty}$ triangular map $F$ from Theorem 5.8, by the theorem of Yomdin 
([Y], cf. [G]) we have

$$
h\left(F ; I_{f(c)}\right) \geq \limsup _{n \rightarrow \infty} \frac{1}{n} \log \operatorname{Vol}\left(\Gamma_{g_{f(c)}^{[n]}}\right),
$$

where $g_{f(c)}^{[n]}=g_{f^{n}(c)} \circ \ldots \circ g_{f^{2}(c)} \circ g_{f(c)}$. We know (see Example 5.2) that

$$
\operatorname{Vol}\left(\Gamma_{\left.g_{f(c)}^{\left[{ }^{n}\right.}-1\right]}\right)=\operatorname{Vol}\left(\Gamma_{\varphi_{1}^{2^{n}-1}}\right)=\operatorname{Vol}\left(\Gamma_{\eta_{1}^{a_{n}}}\right) \geq 2^{a_{n}}
$$

Thus

$$
h(F) \geq h\left(F ; I_{f(c)}\right) \geq \limsup _{n \rightarrow \infty} \frac{1}{2^{n}-1} \log 2^{a_{n}}=\frac{1}{2} \log 2>0 .
$$

\section{References}

[AKM] R. L. Adler, A. G. Konheim and M. H. McAndrew, Topological entropy, Trans. Amer. Math. Soc. 114 (1965), 309-319.

[ALM] L. Alsedà, J. Llibre and M. Misiurewicz, Combinatorial Dynamics and Entropy in Dimension One, World Sci., Singapore, 1993.

[BEL] F. Balibrea, F. Esquembre and A. Linero, Smooth triangular maps of type $2^{\infty}$ with positive topological entropy, Internat. J. Bifur. Chaos 5 (1995), $1319-1324$.

[BC] L. S. Block and W. A. Coppel, Dynamics in One Dimension, Lecture Notes in Math. 1513, Springer, Berlin, 1992.

[B] R. Bowen, Entropy for group endomorphisms and homogeneous spaces, Trans. Amer. Math. Soc. 153 (1971), 401-414.

[CE] P. Collet and J.-P. Eckmann, Iterated Maps on the Interval as Dynamical Systems, Progr. in Phys. 1, Birkhäuser, Boston, 1980.

[D] E. I. Dinaburg, Connection between various entropy characterizations of dynamical systems, Izv. Akad. Nauk SSSR 35 (1971), 324-366 (in Russian).

[G] M. Gromov, Entropy, homology and semialgebraic geometry (after Y. Yomdin), Astérisque (Séminaire Bourbaki, 1985-86, no. 663) 145-146 (1987), 225-240.

[Ka] A. Katok, Lyapunov exponents, entropy and periodic orbits for diffeomorphisms, Publ. Math. IHES 51 (1980), 137-174.

[Kl] P. E. Kloeden, On Sharkovsky's cycle coexistence ordering, Bull. Austral. Math. Soc. 20 (1979), 171-177.

[Ko] S. F. Kolyada, On dynamics of triangular maps of the square, Ergodic Theory Dynam. Systems 12 (1992), 749-768.

[KS] S. Kolyada and L. Snoha, Topological entropy of nonautonomous dynamical systems, Random Comput. Dynam. 4 (1996), 205-233.

[dMvS] W. de Melo and S. van Strien, One-Dimensional Dynamics, Series of Modern Surveys in Math., Springer, Berlin, 1993.

[M] M. Misiurewicz, Attracting set of positive measure for a $C^{\infty}$ map of an interval, Ergodic Theory Dynam. Systems 2 (1982), 405-415.

[MS] M. Misiurewicz and W. Szlenk, Entropy of piecewise monotone mappings, Studia Math. 67 (1980), 45-63. 
[Y] Y. Yomdin, Volume growth and entropy, Israel J. Math. 57 (1987), 285-300.

Institute of Mathematics

Ukrainian Academy of Sciences

Tereshchenkivs'ka 3

$252601 \mathrm{Kiev}$, Ukraine

E-mail: skolyada@imath.kiev.ua

Department of Mathematical Sciences

IUPUI

402 N. Blackford Street

Indianapolis, Indiana 46202-3216

U.S.A

E-mail: mmisiure@math.iupui.edu
Department of Mathematics Faculty of Natural Sciences Matej Bel University Tajovského 40

97401 Banská Bystrica, Slovakia E-mail: snoha@fpv.umb.sk

Received 11 November 1998;

in revised form 27 January 1999 